\title{
A atratividade facial percebida por leigos e cirurgiões-dentistas: uma revisão sistemática
}

\author{
Facial attractiveness perceived by lay people and dentists: \\ a systematic review
}

\author{
José Henrique Saldanha* \\ Anderson Paulo Barbosa Lima** \\ Camila Vas Tostes Mendes* \\ Tadeu Evandro Mendes Junior ${ }^{* * *}$ \\ Tadeu Evandro Mendes ${ }^{* * * *}$ \\ Luiz Renato Paranhos ${ }^{* * * *}$
}

\section{Resumo}

Objetivo: avaliar sistematicamente, com base na literatura, se a opinião de leigos é mais flexível na avaliação da estética facial quando comparada à opinião de cirurgiões-dentistas. Materiais e método: foram utilizadas para a pesquisa as bases de dados eletrônicas LILACS, PubMed, SciELO e OpenGrey. Especificamente, a base OpenGrey foi utilizada para captação da "literatura cinza". Foram selecionados artigos publicados nos últimos dez anos, com textos completos disponíveis on-line em inglês, espanhol e português. Os artigos indexados em mais de uma base de dados foram contabilizados somente uma vez. Foram excluídos os artigos de revisão da literatura, relatos de caso e editoriais/cartas ao editor. Toda a pesquisa foi feita por dois revisores de elegibilidade. Resultados: foram identificados 118 registros, tendo sido três estudos elegíveis para a pesquisa. Todos os trabalhos selecionados envolviam ortodontistas e leigos como avaliadores. A Escala Analógica Visual e um Escore de Avaliação de Perfil foram utilizados como mensuração, analisados pelas fotografias. Dois artigos analisaram alterações anteroposteriores e um artigo analisou alterações na assimetria facial (frontal). Conclusão: mediante os estudos avaliados, nota-se que não há evidências claras de que leigos são mais flexíveis nas avaliações da estética facial.

Palavras-chave: Dentista. Estética. Face. Ortodontia. Percepção.

\section{Introdução}

A perspectiva atual do tratamento ortodôntico está, constantemente, ligada à estética do indivíduo, que pode variar de acordo com vários fatores. Devido ao caráter subjetivo dos parâmetros estéticos, faz-se necessário um consenso entre profissional e paciente para se chegar a objetivos comuns no tratamento ${ }^{1}$.

Um estudo ${ }^{2}$ mostra que as características de um perfil côncavo combinado com aumento vertical inferior da face são percebidas como menos atraentes. Da mesma maneira, indivíduos com perfil reto e com proporções verticais equilibradas são percebidos como mais atraentes ${ }^{3}$, enquanto indivíduos com perfil convexo são menos atraentes, principalmente os do gênero masculino ${ }^{4}$. Lábio leporino, microssomia hemifacial e fratura da mandíbula na infância são fatores associados à assimetria facial grave ${ }^{5}$. Em contrapartida, uma menor assimetria facial, determinada como a diferença da hemiface direita e esquerda ou assimetria normal, é mais habitual ${ }^{6}$. Essa percepção é determinada pelo senso clínico do cirurgião-dentista e do indivíduo ${ }^{7}$.

Cirurgiões-dentistas em clínica privada, Varginha, MG, Brasil.

Mestrando em Ortodontia, Centro de Ciências da Saúde, Universidade do Sagrado Coração, Bauru, SP, Brasil.

Mestrando em Ciências Odontológicas, Faculdade de Odontologia, Universidade de São Paulo, São Paulo, SP, Brasil.

Professor, Instituto Nacional de Ensino Superior e Pós-Graduação Padre Gervásio, Varginha, MG, Brasil.

Professor Adjunto, Departamento de Odontologia, Universidade Federal de Sergipe, Lagarto, SE, Brasil. 
A avaliação da face do paciente é uma manobra importante na elaboração do diagnóstico e do plano de tratamento. O profissional deve seguir suas percepções e atender às expectativas funcionais e estéticas do paciente. Dessa forma, o presente estudo objetiva comparar, por meio de uma revisão sistemática da literatura, a percepção da atratividade da análise facial na opinião de leigos e de cirurgiões-dentistas.

\section{Materiais e método}

\section{Questão norteadora e critérios de elegibilidade}

Foram selecionados estudos sobre a percepção da estética facial entre leigos e cirurgiões-dentistas. A questão da pesquisa foi baseada na estratégia PICO (P - Polpulação; I - Introdução; C - Comparação; O - Desfecho [Outcome]). Critérios de inclusão e exclusão estão esclarecidos na Tabela 1.

Tabela 1 - Descrição da estratégia de busca utilizada na pesquisa

\section{Estratégia de busca}

Pergunta norteadora: os leigos são mais condescendentes na avaliação da estética facial quando comparados com cirurgiões-dentistas?

\begin{tabular}{|c|c|}
\hline Componentes da estratégia PICO & Descrição \\
\hline População & Indivíduos tratados ou não pela terapia ortodôntica. \\
\hline Intervenção & Avaliação da atratividade facial. \\
\hline Comparação & Comparação da percepção estética por leigos e por cirurgiões-dentistas. \\
\hline Desfecho & Divergência ou não na análise da atratividade facial. \\
\hline Bases de dados eletrônicas & LILACS, PubMed, SciELO e OpenGrey \\
\hline Critérios de elegibilidade & $\begin{array}{l}\text { Inclusão: foram selecionados os artigos que tivessem somente cirurgiões-dentistas } \\
\text { (formados) e leigos como avaliadores, para convergir com os objetivos do presente } \\
\text { trabalho; textos completos on-line em inglês, português ou espanhol; apenas trabalhos } \\
\text { dos últimos dez anos. } \\
\text { Exclusão: foram excluídos trabalhos de revisão de literatura, opiniões, relatos de casos; } \\
\text { editorial e/ou carta ao editor; estudos que não avaliavam fotografias extrabucais. }\end{array}$ \\
\hline
\end{tabular}

\section{Estratégia de pesquisa para identificação de estudos}

Os seguintes descritores foram selecionados junto ao MeSH (PubMed): percepção, dentista, face, ortodontista e estética. Foram empregadas as expressões booleanas and e or para realizar as combinações. Foram utilizadas quatro bases de dados eletrônicas. Especificamente, a OpenGrey foi utilizada para buscar a "literatura cinza" acerca do tema e evitar vieses de seleção e publicação (Tabela 1). A pesquisa foi realizada no mês de abril de 2015.

A primeira fase da identificação dos estudos foi realizada pela análise dos títulos e resumos por dois revisores de elegibilidade (JHVS e APBL), que trabalhavam de forma independente, sem sigilo para o nome dos autores e das revistas. A Tabela 2 mostra a estratégia de busca utilizada. 


\begin{tabular}{|c|c|}
\hline Base de dados & Estratégia de pesquisa \\
\hline $\begin{array}{l}\text { PubMed } \\
\text { http://www.ncbi.nlm.nih.gov/pubmed }\end{array}$ & $\begin{array}{l}\text { ("esthetics"[MeSH Terms] OR "esthetics"[All Fields] OR "esthetic"[All Fields]) AND } \\
\text { ("perception"[MeSH Terms] OR "perception"[All Fields]) AND ("orthodontics"[MeSH } \\
\text { Terms] OR "orthodontics"[All Fields]) } \\
\text { ("face"[MeSH Terms] OR "face"[All Fields]) AND ("perception"[MeSH Terms] OR } \\
\text { "perception"[All Fields]) AND ("dentistry"[MeSH Terms] OR "dentistry"[AII Fields]) } \\
\text { ("face"[MeSH Terms] OR "face"[All Fields]) AND ("perception"[MeSH Terms] OR } \\
\text { "perception"[All Fields]) AND ("orthodontics"[MeSH Terms] OR "orthodontics"[AII } \\
\text { Fields]) }\end{array}$ \\
\hline $\begin{array}{l}\text { LILACS } \\
\text { http://lilacs.bvsalud.org }\end{array}$ & $\begin{array}{l}\text { "Face" AND "Perception" AND "Dentistry" } \\
\text { "Esthetics" AND "Perception" AND "Orthodontics" } \\
\text { "Face" AND "Perception" AND "Orthodontics" }\end{array}$ \\
\hline $\begin{array}{l}\text { SciELO } \\
\text { http://www.scielo.org }\end{array}$ & $\begin{array}{l}\text { "Face" AND "Perception" AND "Dentistry" } \\
\text { "Esthetics" AND "Perception" AND "Orthodontics" } \\
\text { "Face" AND "Perception" AND "Orthodontics" }\end{array}$ \\
\hline $\begin{array}{l}\text { OpenGrey } \\
\text { www.opengrey.eu }\end{array}$ & $\begin{array}{l}\text { "Face" AND "Perception" AND "Dentistry" } \\
\text { "Esthetics" AND "Perception" AND "Orthodontics" } \\
\text { "Face" AND "Perception" AND "Orthodontics" }\end{array}$ \\
\hline
\end{tabular}

Fonte: dados da pesquisa.

Para os estudos incluídos preliminarmente e para aqueles que não apresentavam, no título e no resumo, dados suficientes para tomar uma decisão clara, os textos completos foram obtidos e avaliados para determinar se contemplavam todos os critérios de elegibilidade. Estudos recusados em qualquer fase foram registrados em uma tabela de exclusão, na qual os motivos foram anotados. Em casos em que restaram dúvidas, foram solicitados esclarecimentos por e-mail.

\section{Qualidade individual dos estudos}

Os textos completos de todos os artigos elegíveis foram avaliados pela qualidade metodológica, utilizando o check-list adaptado de Cericato et al. ${ }^{8}$ (2015), por dois revisores de elegibilidade (JHVS e APBL). O escore de avaliação variou de 0 a 10 pontos. Os estudos foram classificados como sendo de baixa (nota de 0 a 4), moderada (nota de 5 a 7 ) ou alta (nota 8 a 10) qualidade metodológica. Os estudos considerados de baixa qualidade foram tidos como metodologicamente pobres, tendo sido descartados. Nesse momento, os revisores estavam cegos para os autores e revistas, evitando qualquer viés de seleção e possíveis conflitos de interesse. Caso houvesse discordância, um terceiro examinador (LRP) era consultado.

\section{Extração e análise dos dados}

Depois da triagem, os textos completos dos artigos selecionados foram reanalisados, utilizando-se uma ficha padronizada de extração de dados, em que se verificou a autoria, o ano de publicação, o grupo avaliado, os avaliadores, o método e os resultados. Os dados foram coletados por meio de uma análise descritiva dos estudos selecionados, sendo o produto final da avaliação apresentado de forma narrativa/dissertativa.

\section{Resultados}

\section{Estratégia de pesquisa}

$\mathrm{Na}$ pesquisa realizada nas bases de dados eletrônicas LILACS ${ }^{\circledR}$, PubMed $^{\circledR}$, SciELO $^{\circledR}$ e OpenGrey $^{\circledR}$, foram identificados 118 registros, dos quais 15 foram excluídos por apresentarem duplicidade. Dos 103 registros restantes, 100 foram excluídos por não estarem em conformidade com a estratégia de pesquisa utilizada no presente trabalho, de modo que apenas três artigos foram considerados elegíveis para o estudo (Figura 1). 


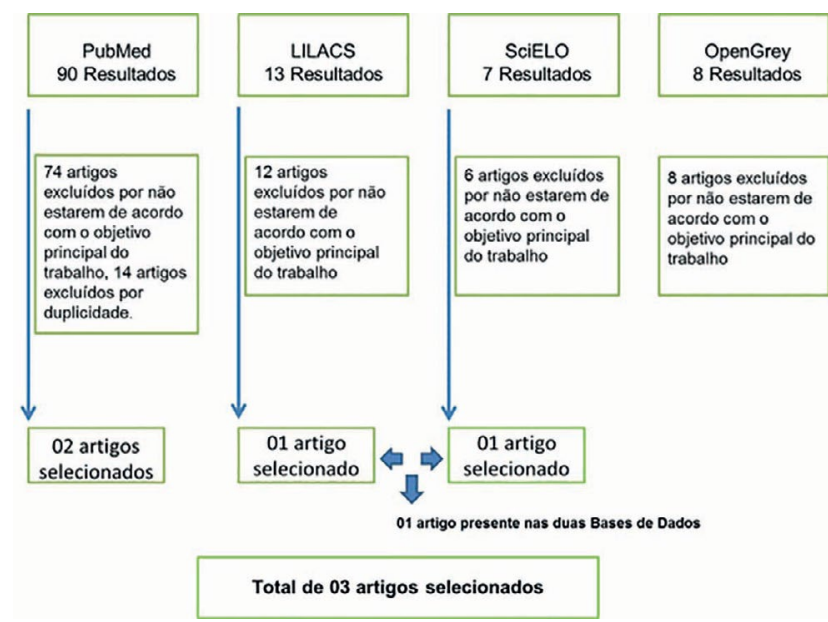

Figura 1 - Fluxograma com a seleção dos artigos e os critérios utilizados Fonte: dados da pesquisa.

\section{Qualidade individual dos estudos}

$\mathrm{Na}$ avaliação da qualidade dos estudos, realizada de acordo com os critérios adaptados de Cericato et al. ${ }^{8}$ (2015), nenhum artigo foi reprovado por "baixa qualidade" metodológica (escore de 0 a 4 ).

\section{Estudos incluídos}

Os três artigos elegíveis apresentaram comparações entre a percepção da normalidade facial entre leigos e cirurgiões-dentistas em indivíduos tratados ou não. Todos os trabalhos envolveram a especialidade de Ortodontia e utilizaram fotografias para avaliação, comparando erros sagitais ou transversais da face (Tabela 3 ).

Tabela 3 - Sumário das principais características descritivas dos estudos incluídos

\begin{tabular}{|c|c|c|c|c|}
\hline $\begin{array}{l}\text { Autoria, ano e } \\
\text { local do estudo }\end{array}$ & Grupo avaliado & Avaliadores & Método & Desfecho \\
\hline $\begin{array}{l}\text { Fabré et al.² } \\
(2009) \\
\text { Suíça }\end{array}$ & $\begin{array}{l}10 \text { homens e } 8 \text { mulheres } \\
\text { caucasianos, perfil cônca- } \\
\text { vo; } 4 \text { homens e } 5 \text { mulheres } \\
\text { perfil reto como referência. }\end{array}$ & $\begin{array}{l}18 \text { ortodontistas } \\
\text { e } 18 \text { leigos }\end{array}$ & $\begin{array}{l}\text { Fotografias coloridas de } \\
\text { perfil de ambos os grupos }\end{array}$ & $\begin{array}{l}\text { Os leigos foram menos } \\
\text { críticos na avaliação } \\
\text { dos perfis côncavos e } \\
\text { retos. }\end{array}$ \\
\hline $\begin{array}{l}\text { Sattarzadeh e } \text { Lee }^{10} \\
(2010) \\
\text { Inglaterra }\end{array}$ & $\begin{array}{l}9 \text { homens entre } 12-14 \text { anos } \\
\text { e } 7 \text { mulheres entre } 11-13 \\
\text { anos com perfil convexo }\end{array}$ & $\begin{array}{l}34 \text { ortodontistas } \\
\text { e } 35 \text { leigos }\end{array}$ & $\begin{array}{l}32 \text { fotografias digitaliza- } \\
\text { das do pré e pós-trata- } \\
\text { mento com terapia Twin } \\
\text { Block }\end{array}$ & $\begin{array}{l}\text { Não houve diferença } \\
\text { significativa entre as } \\
\text { avaliações de ortodon- } \\
\text { tistas e leigos. }\end{array}$ \\
\hline $\begin{array}{l}\text { Silva et al. }{ }^{9} \\
(2011) \\
\text { Brasil }\end{array}$ & $\begin{array}{l}1 \text { homem e } \\
1 \text { mulher com perfil reto }\end{array}$ & $\begin{array}{l}30 \text { ortodontistas } \\
\text { e } 30 \text { leigos }\end{array}$ & $\begin{array}{l}4 \text { fotografias: } 1 \text { em } \mathrm{MIH} \mathrm{e} \\
3 \text { com desvios mandibu- } \\
\text { lares de } 2 ; 4 ; 6 \mathrm{~mm} \text {. }\end{array}$ & $\begin{array}{l}\text { Os ortodontistas são } \\
\text { mais críticos com assi- } \\
\text { metrias severas. }\end{array}$ \\
\hline
\end{tabular}

Fonte: dados da pesquisa.

Fabré et al. ${ }^{2}$ utilizaram um escore de avaliação de perfil (Profile Assessment Score - PAS) de 0 a 10 pontos (0 perfil pouco atraente e 10 perfil muito atraente) e mostraram que leigos e ortodontistas consideraram indivíduos de perfil reto mais atraentes que indivíduos de perfil côncavo e que, ao avaliar perfis côncavos, os leigos são mais condescendentes que os ortodontistas, mesmo sem diferenças estatisticamente significativas. Os autores observaram que o grau da concavidade facial e a inclinação do plano mandibular foram fatores determinantes para o baixo escore na avaliação de perfil dos indivíduos.

Sattarzadeh e Lee ${ }^{10}$ utilizaram escala VAS e não detectaram diferenças relevantes nas avaliações pré-tratamento de indivíduos com perfil convexo, no entanto, foram identificadas notas mais elevadas para as avaliações realizadas pós-tratamento, mas que não mostram uma diferença estatisticamente significativa. Os autores identificaram que ortodontistas foram hábeis em observar desvios a partir de $4 \mathrm{~mm}$ comparados com Máxima Intercuspidação Habitual (MIH), para ambos os gêneros, enquanto os leigos observaram da mesma maneira indivíduos do gênero feminino, mas não observaram alterações a partir de MIH no gênero masculino.

Silva et al. ${ }^{9}$ utilizaram a Escala Visual Analógica (Visual Analogue Scale - VAS) de 0 a 10 pontos e obtiveram notas mais críticas para pacientes do gênero feminino. Mostraram que os ortodontistas tendem a ser mais críticos em assimetrias severas que os leigos, mas sem diferenças estatisticamente significativas $(\mathrm{p}>0,05)$.

\section{Discussão}

O tema da beleza tem sido muito discutido ao longo da história, e estratégias para a avaliação da beleza têm sido o âmago de muitos estudos ${ }^{11,12}$. $\mathrm{O}$ uso de imagens alteradas por software simulando as alterações da face auxilia o diagnóstico e o plano de tratamento entre paciente, pais e cirurgião-dentista, pois se o ortodontista utilizar somente telerradiografia lateral da face como método de diagnóstico, ele deixará de lado as alterações de tecido mole ${ }^{13}$.

A avaliação da estética facial depende muito da percepção de quem e da forma de como se observa. 
É influenciada por fatores como gênero, raça e meio social ${ }^{11}$. Com a finalidade de uniformizar os resultados desta pesquisa, foram incluídos apenas os artigos que utilizaram fotografias para a avaliação da estética facial. Embora outras pesquisas abordem a avaliação da percepção estética da face por meio de silhuetas com a finalidade de eliminar fatores extrínsecos, desviando os objetivos da pesquisa, acredita-se que a utilização de fotografias traduz mais fielmente a realidade.

Os estudos de Silva et al. ${ }^{9}$ e de Sattarzadeh e Lee ${ }^{10}$ não apresentaram diferença estatisticamente significativa da percepção dos avaliadores, medida pela escala VAS. Fabré et al. ${ }^{2}$, utilizando PAS, também não encontraram diferenças estatisticamente significativas - os resultados foram bastante semelhantes. A escala de avaliação parece não influenciar na mensuração da percepção da estética facial.

Leigos deram pontuação um pouco mais elevada do que cirurgiões-dentistas (ortodontistas) na avaliação transversal ${ }^{9}$ e sagital ${ }^{2,10}$ da face. Na análise transversal da face, a assimetria foi percebida em desvios de $4 \mathrm{~mm}$ a $6 \mathrm{~mm}$ (ortodontistas) e acima de $6 \mathrm{~mm}$ (leigos). Os indivíduos da amostra do gênero feminino e masculino tiveram notas semelhantes avaliados por leigos, entretanto, na avaliação dos ortodontistas, o gênero feminino teve menores pontuações ${ }^{9}$. A percepção de leigos e ortodontistas tende a ser semelhante na avaliação da face normal e diferir à medida que a assimetria mandibular vai aumentando 9 . Esse resultado ficou mais evidente na amostra do gênero feminino, mesmo não sendo estatisticamente significativa ${ }^{9}$.

A avaliação da concavidade facial obteve notas mais baixas, demonstrando atrair mais atenção negativamente no quesito estética facial. A inclinação do plano mandibular juntamente com a concavidade facial, comparadas com perfil normal, demonstrou ser menos atraente para leigos e ortodontis$\operatorname{tas}^{2}$. No grupo dos avaliadores do perfil côncavo e do perfil normal, a faixa etária era distinta e parece não ter influenciado nos resultados. Mesmo com resultados não significativos, leigos demonstram ser mais tolerantes com discrepâncias esqueléticas em comparação a ortodontistas ${ }^{2}$. Esses achados podem justificar que, frequentemente, cirurgiões-dentistas percebem aspectos faciais que podem ser irrelevantes para leigos.

Em um estudo, os autore ${ }^{10}$ concluíram que leigos e ortodontistas não avaliaram a convexidade facial (com pelo menos $7 \mathrm{~mm}$ de overjet) como pouco atraente. Pontuações superiores foram dadas pelo grupo dos leigos em comparação ao grupo dos ortodontistas, mas a diferença não foi estatisticamente significativa $^{10}$. Na avaliação de alteração sagital da face, leigos demonstraram ser mais complacentes do que os ortodontistas, corroborando o estudo de Fabré et al. ${ }^{2}$.

Dessa forma, para um melhor diagnóstico, a avaliação das medidas cefalométricas da face e das as- simetrias deve ser sempre observada e questionada com o paciente, pois suas queixas e seus objetivos, e não apenas a percepção do profissional, devem ser levados em consideração durante todo o tratamento. Assim, os profissionais da saúde (principalmente o cirurgião-dentista) não devem se ater em seguir apenas as suas percepções treinadas, e sim respeitar cada vez mais a opinião dos leigos no momento do diagnóstico. Deve-se almejar não somente dentes bem posicionados, mas uma face esteticamente agradável no meio social em que o paciente convive.

\section{Conclusão}

Mediante os estudos avaliados, nota-se que não há evidências claras de que leigos são mais flexíveis nas avaliações da atratividade facial quando comparados aos cirurgiões-dentistas (ortodontistas).

\section{Abstract}

Objective: To systematically assess by evidence-based literature whether lay people are more flexible in evaluating facial aesthetics when compared to dentists. Materials and method: LILACS, PubMed, SciELO, and OpenGrey electronic databases were used for the research. OpenGrey database was particularly used to capture the "grey literature". Articles from the last 10 years with full texts available online in English, Spanish, and Portuguese were selected. The articles indexed in more than one database were computed only once. Literature review articles, case reports, and editorial / letters to the editor were excluded. The entire research was performed by two eligibility reviewers. Results: 118 records were identified, and three studies were eligible for the research. All works selected involved orthodontists and lay people as evaluators. The Visual Analogue Scale and a Profile Assessment Score were used for measurement and analyzed by photographs. Two articles analyzed anteroposterior changes and one article analyzed changes in facial asymmetry (frontal). Conclusion: Given the studies evaluated, it is imposed there is no clear evidence that lay people are more flexible in assessing facial aesthetics.

Keywords: Perception. Dentist. Orthodontics. Face. Aesthetics.

\section{Referências}

1. Reis SAB, Abrão J, Capelozza Filho L, Claro CAA. Análise facial subjetiva. Rev Dental Press Ortodon Ortop Facial 2006; 11(5):159-72.

2. Fabré M, Mossaz C, Christou P, Kiliaridis S. Orthodontists' and laypersons' aesthetic assessment of Class III subjects referred for orthognathic surgery. Eur J Orthod 2009; 31(4):443-8.

3. Johnston CJ, Hunt O, Johnston CD, Burden DJ, Stevenson $\mathrm{M}$, Hepper P. The influence of lower face vertical proportion on facial attractiveness. Eur J Orthod 2005; 27(4):349-54. 
4. Von Bremen J, Erbe C, Pancherz H, Ruf S. Facial-profile attractiveness changes in adult patients treated with the Herbst appliance. J Orofac Orthop 2014; 75(3):167-74.

5. Haraguchia S, Iguchib Y, Takadac K. Asymmetry of the face in orthodontic patients. Angle Orthod 2008; 78(3):421-6.

6. Good S, Edler R, Wertheim D, Greenhill D. A computerized photographic assessment of the relationship between skeletal discrepancy and mandibular outline asymmetry. Eur J Orthod 2006; 28(2):97-102.

7. Zaidel DW, Cohen JA. The face, beauty, and symmetry: perceiving asymmetry in beautiful faces. Inst J Neurosc 2005; 115(8):1165-73.

8. Cericato GO, Bittencourt MAV, Paranhos LR. Validity of the assessment method of skeletal maturation by cervical vertebrae: a systematic review and meta-analysis. Dentomaxillofac Radiol 2015; 44(4):20140270.

9. Silva NCF, Aquino ERB, Mello KCFR, Mattos JNR, Normando D. Habilidade de ortodontistas e leigos na percepção de assimetrias da mandíbula. Dental Press J Orthod 2011; 16(4):38.e1-8

10. Sattarzadeh AP, Lee RT. Assessed facial normality after Twin Block therapy. Eur J Orthod 2010; 32(4):363-70.

11. White MJ, Evans CA, Viana G, Anderson NK, Giddon DB. Facial profile preferences of black women before and after orthodontic treatment. Am J Orthod Dentofacial Orthop 2006 ; 129(1):17-23.

12. Yu X, Liu B, Pei Y, Xu T. Evaluation of facial attractiveness for patients with malocclusion: a machine-learning technique employing Procrustes. Angle Orthod 2014; 84(3):410-6.

13. Miner RM, Anderson NK, Evans CA, Giddon DB. The perception of children's computer-imaged facial profiles by patients, mothers and clinicians. Angle Orthod 2007; 77(6):1034-9.

\section{Endereço para correspondência:}

Luiz Renato Paranhos

Rua Jordão de Oliveira, 996, apart. 1.402

Bairro Atalaia

49037-330 Aracaju, SE

Telefone: (79) 991161896

E-mail: paranhos.Irp@gmail.com

Recebido: 20/01/16. Aceito: 23/03/16. 